\title{
On the Empirical Limits of Billboard Rotation
}

\author{
Elodie Fourquet* William Cowan ${ }^{\dagger} \quad$ Stephen Mann ${ }^{\ddagger}$ \\ David R. Cheriton School of Computer Science, University of Waterloo, Canada
}

\begin{abstract}
This paper describes an experiment investigating for the first time, the perceptual tolerance of inconsistent projections when rotated billboards are transformed and included in an image among other objects produced by traditional 3D computer graphics. The need for this results has arisen in building a system to assist artists with simultaneous composition in two and three dimensions. Imitating the cartoon-based compositional practices of Renaissance artists we are using billboards as modelling primitives, with the additional freedom of manipulating them in three dimensions rather than two. Three-dimensional manipulation adds the capability of rotating billboards about vertical axes, which introduces geometric distortions exactly like those produced by multiple projections in Renaissance painting.
\end{abstract}

An experiment measured the limits of acceptable distortion by having subjects choose the distorted object from an array of undistorted ones. The results include a concrete limit for acceptable billboard rotations, an identification of object features that create exceptional limits to rotation, and some conclusions about the relationship of canonical views to billboard use.

CR Categories: I.3.3 [Computer Graphics]: Picture/Image Generation- [I.3.7]: Computer Graphics-Three-Dimensional Graphics and Realism J.4 [Social and Behavioral Sciences]: Psychology-

Keywords: perception, psychophysics, billboard, projection, rotation, geometric transformation, silhouette

\section{Introduction}

This paper is about the perception of two-dimensional pictures with three-dimensional content, positioned and transformed in a scene. They are viewed two and three dimensionally at the same time: viewers respond to the three-dimensional placement of objects in the picture, and also to two-dimensional arrangements of objects in the picture plane. Producing a satisfactory compromise between these two competing objectives is impossible without relaxing 'realism'. Realist painters have long known this. In Renaissance painting, for example, artists knew that unitary perspective is largely unnecessary, and sometimes even detrimental. For pictorial composition they used collages of cartoons, each having its own local projection, and the ensuing geometric inconsistencies go largely

\footnotetext{
*e-mail: efourque@cgl.uwaterloo.ca

†e-mail: wmcowan@cgl.uwaterloo.ca

‡e-mail: smann@cgl.uwaterloo.ca
}

unnoticed, especially for objects like human figures. That is, the painters discovered relaxations of realism that, particularly for human figures, increase compositional flexibility without creating objectionable geometrical inconsistencies. Of course, the informal, 'by eye' methods of artists are not amenable to computation. A substructure of understanding in perception and computer graphics is needed, of which this paper supplies a small part.

Perception is a seamless construction of sensation and memory, which is the root of the universal observation that familiarity, habit and context are essential components of what is perceived. Early artists, in Egypt for example, showed each object in its most familiar orientation, a practice that is subtly continued in later realistic art, where aspects of the familiar view are over-emphasized if it is necessary to show an object away from its habitual orientation. Feet, for example, are most often seen from above, which emphasizes the toes and widens the foot. In Renaissance painting, when a painted figure is shown facing the viewer the feet are shown pointing down, to display the toes, and foreshortening is exaggerated.

These 'unrealistic' modifications actually make the image more realistic. Why? Normal vision is active: the viewer seeks information from many different points of view and puts it all together in the brain. This ability is unavailable when viewing an image, and the artist compensates by exaggerating cues that duplicate information normally picked up by active perception. The result is an artistic preference for what psychologists call the 'canonical view' [Palmer et al. 1981], another constraint on composition.

Issues of artistic depiction, like the ones discussed above, are currently important in computer graphics because of the ubiquity of billboards and other image-based techniques, which transfer twodimensional depictions directly into the picture. This approach is highly efficient, and able to introduce non-computer-originated imagery into a picture, but at the cost of unpredictable geometrical distortions, 'unpredictable', because without extensive experimentation it is impossible to know how human viewers respond to the distortions. The comments above suggest that billboards may have another, unforeseen, application as modelling primitives, bridging the divide between two-dimensional illustration tools like Illustrator or Photoshop and full three-dimensional modelling environments, or as a generalized form of compositing.

As modelling primitives billboards are translated in three dimensions, scaled and rotated. Only translation and scaling are extensively used with billboards at present because their geometry is so well understood that practitioners can follow their intuitions when judging distortion. Rotation, however, is a different matter, with billboard use restricted to a few standard orientations, parallel to the view plane or perpendicular to the eye ray, and/or to objects, like trees and spheres, that have strong, if approximate, symmetries.

Engaged in an extended study of billboards as modelling primitives, we find billboard rotation greatly extends the scope and flexibility of modelling. Thus we have undertaken the present experiment, which measures the visibility of geometric distortion that occurs when billboards are rotated as an approximation to object rotation. In addition to advancing our research project, the results extend the range of phenomena amenable to billboard rendering. More profoundly, they begin to delimit the acceptability of multiple projections within a single image, which both enlarges the potential content of billboards and the ways in which they can be used. 


\section{Related Work and Motivation}

Billboards are an attractive modelling concept because their computation cost is independent of their content. Thus, it is unsurprising that they have a long history in computer graphics, though under a variety of names. For example, billboards, called sprites and implemented in hardware, existed in the TI9918A graphics controller as early as 1980. Hardware implementation of basic billboard algorithms, most of which are conceptually similar to texture-mapping, continues: it is only a slight stretch to regard shaders, which lie at the heart of GPU rendering, as algorithmic billboards.

With hardware support increasing the efficiency of billboards, recent research freely multiplies billboards as a method of making them applicable to new subject matter. Thus, Jeschke et al. [2005], who follow Maciel and Shirley [1995] in calling billboards impostors, review many methods for capturing the appearance of complex subject matter using billboard clouds [Décoret et al. 2003]. Yet despite extensive research on finding new algorithms for billboards, there has been little formal evaluation of their perceptual acceptability. To be sure, every paper shows images that demonstrate the acceptability of several instances of billboard content, and the marketing success of video game producers shows that billboards are acceptable for peripheral material in fast-moving scenes, but these measures fail to address quantitative thresholds of acceptability. Thus, the recent experiments of Hamill et al. [2005] are very welcome. They compared images rendered from $3 \mathrm{D}$ data to images rendered from billboards, where the subjects were architecture and animations of moving figures. For these images they measured thresholds below which users were unable to distinguish a difference between the two, in terms of pixel-to-texel ratio for architecture, in terms of minimum perceivable difference in motion for moving figures. This experiment, which maintained billboard orientation, examined parts of the parameter space that are not relevant to our focus on the distortion from inconsistent projection, but the division of subject matter into architecture and human figures is similar to our division between geometric and organic objects.

By contrast, psychological research in picture perception has considered extensively the effects of non-central view direction on the perception of images. The core question in this research is the existence or non-existence of automatic constancy mechanisms that compensate for oblique viewing as distance constancy compensates for differences in retinal size. Cutting [1987] takes one point of view, that geometric distortion due to oblique viewing is negligible, and simply ignored. Busey et al. [1990] qualifies this claim, showing that it holds only for viewing angles less than $22^{\circ}$. More recently, Vishwanath et al. [2005] assert, counter-intuitively, that there exists a mechanism to correct automatically non-centered viewing angle and that it plays an essential role for large viewing angles.

While the objective distortions of picture perception research are similar to the distortions we ask our subjects to identify, the context is very different. When a picture is viewed obliquely all parts of it are viewed in the same projection, consistency giving the viewer many cues for the view direction. In the applications that motivate our research a small part of the picture, the billboard, is viewed non-centrally. The viewer does not know that oblique viewing is present, but may infer it from inconsistencies with other parts of the picture, the inconsistency occurring as geometric distortion. All the same the similarity between the thresholds noticed by Busey et al. and the onset of perceivable distortion in our preliminary experiment [Fourquet et al. 2006] suggests that the dominant perceptual mechanism may be the same in both cases. Reinforcing this idea, the fourth experiment of Busey at al. [1990] showed the frame to play little role in the perception of distortion.
Another aspect of picture perception that is relevant to our interests is the 'canonical view', first so named by Palmer et al. [1981]. It seems that specific object types are best identified from a restricted range of view directions. Blanz et al. [1999] demonstrated that the canonical view varies with type of object. For example, from directly in front a cube is indistinguishable from a flat square, and active vision seeks a second look from a different direction to resolve the ambiguity. With a picture the view direction is set when the picture is created, defeating this strategy of active vision.

We expect canonical views to play a significant role in the visibility of distortion, so we used the results of Blanz et al. [1999] in deciding the best reference angle for the objects used in the experiment, as explained in Section 3.2. The results of the experiment contribute to our understanding of the relationship between distortion and canonical views.

\section{Experiment Design and Implementation}

We have run several experiments to measure human subjects' ability to notice the geometric inconsistencies produced by using billboard rotation as an approximation for rotation of threedimensional objects, the aspect of billboard use that is most likely to limit the potential of billboards as modelling primitives.

Our experiments mimic compositions like 'The School of Athens' of Raphael, where many differently projected images co-exist in one picture, or computer graphics pictures in which billboards and $3 \mathrm{D}$ objects appear together. A stringent measure of visible distortion is a forced choice experiment in which the subject identifies the 'most distorted' object among many, only one of which is differently projected.

This is an example of the 'oddity' or 'odd man out' experiment, a standard psychophysical design [Macmillan and Creelman 1991]. This design, which requires the subject to identify the signal that is unlike the others in some respect, needs at least three signals on which same/different judgments can be made. We used only three signals, which were projected images, to reduce the size of the experiment, which made a complete design possible. It is essential for the experiment to exclude 2D pattern matching. Therefore, the three images are rotated to different orientations before projection, forcing the subject to interpret the images as projections of $3 \mathrm{D}$ objects.

Thus, in our experiment subjects are shown the images of three objects, each rotated from its canonical orientation by a different amount. Their task is to identify the object that is distorted in its $3 \mathrm{D}$ form with respect to the other two, without being able to respond by simple image comparison.

\subsection{Environment}

The experiment was performed in a dimly lit quiet room. The images were displayed on a shadowmask CRT a little too tightly focused for ideal image viewing, but similar to and better than most displays used for presenting computer graphics images. With the subjects seated about $70 \mathrm{~cm}$ from the display, a single pixel on the display subtended about one minute of visual angle.

A Java ${ }^{T M}$ program displayed the images and acquired subjects' responses from the keyboard. The program used full-screen exclusive mode with the painting handled by the application itself without intervention of the AWT paint event. Thus, the rendering loop was fully controlled and exclusive, with double-buffering used to switch display content. Response times in the experiment are several seconds and the differences between conditions are hundreds of milliseconds which is comparable to inter-subject variation, so 

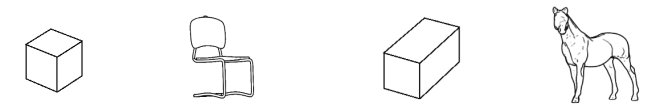

Figure 1: Canonical views for each object, defined $0^{\circ}$ orientation. For the parallelepiped and the horse it is $32^{\circ}$ from the long axis, and for the cube and the chair it is $42^{\circ}$ from the faces.

ordinary Java timing, which has inherent noise of about ten milliseconds, is adequately precise for the experiment.

\subsection{Stimuli}

Each stimulus consisted of three images side by side, as if they were positioned on a common ground plane. The images all represented the same object, rotated about the vertical axis by $30^{\circ}, 0^{\circ}$ and $-30^{\circ}$. (These angles were randomly jittered by $\pm 3^{\circ}$ to diminish the unlikely possibility of subjects holding complete images in their iconic memories.) All three rotation angles were present in every stimulus, randomized among object positions. Two of the images were created by rotating a three dimensional representation of the object about a vertical axis, then perspective projecting it onto the view plane; the other was created by perspective projecting the object onto a billboard, then rotating the billboard into the appropriate orientation, as explained in Section 3.3.

Four different objects were used: a cube, a chair, a parallelepiped and a horse. The canonical views, defining the $0^{\circ}$ orientation for each object, are shown in Figure 1.

The first object is a cube, which has a geometry with which subjects are highly familiar. Its canonical view shows three faces with the view direction slightly away from $45^{\circ}$ so that the vertical sides are slightly unequal in width. Views in which only two faces are shown are deliberately avoided: their ambiguity normally triggers viewpoint change in $3 \mathrm{D}$ vision and they are normally avoided in artistic depiction. The second object is a chair, which shares much of the cube geometry and its canonical view was chosen similarly. Cubes are regular in both side lengths and corner angles. The chair is regular in its side lengths but not its corner angles. Therefore, we used a parallelepiped, which is regular in its corner angles and not its side lengths. Its canonical view, like its side lengths, were chosen to match those of the horse. The horse was chosen because it lacks regularity in angles and lengths: in fact, like most natural objects it lacks defining angles and straight edges. Its canonical view is away from its longitudinal axis, to show side and front views. A comparison of the horse and parallelepiped shows the complexity of canonical views: the parallelepiped has dimensions roughly proportional to those of the horse's body, and the view directions are the same. But the horse, seems more in profile, with the body unnaturally short. Stubbs' paintings show how hard it is to depict a horse, getting its proportions correct.

Blanz et al. [1999] demonstrates that equally canonical views exist over a wide range of rotations. We took advantage of this freedom to avoid non-canonical projections in all the different orientations that occur in the experiment. All orientations in the experiment are measured from the canonical view direction.

The chair and horse images were produced from meshes with 23, 600 and 96, 964 triangles respectively. The meshes were rotated to the correct orientation, after which the DeCarlo suggestive contour algorithm [DeCarlo et al. 2003] was used to create line drawings on the view plane, with hidden lines removed and interior

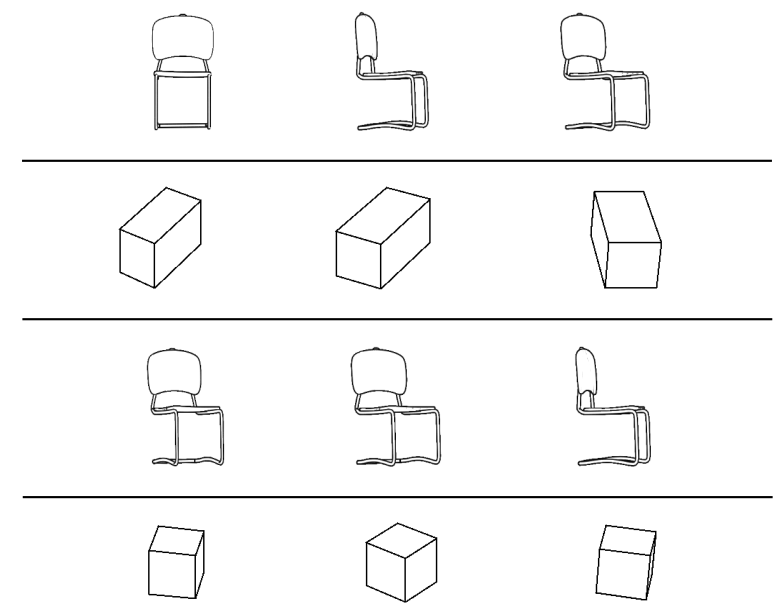

Figure 2: Four stimuli examples. The top two rows contains noticeable distortion. (The billboard is in the left in the top stimuli, and on the left in the second one). The last two rows, presenting a stimuli of the chair and of the cube, have billboard botn on the left.

contours included. The cube and parallelepiped were created as geometric objects, defined by the positions of their corners. They were chosen as objects qualitatively different from the chair and horse in that they have well-defined edges, the alignment of which is easy to perceive. The suggestive contour algorithm was not used because these geometric figures have no interior contours, and it is easy to do hidden line removal explicitly. Typical stimuli examples are shown in Figures 2 and 7.

Initially, in the first part of the experiment, only the cube was used, with the idea that the chair, having roughly cubic contours, would be intermediate between the horse and cube. When it became obvious that the chair and horse clustered away from the cube we deemed it important in the second part of the experiment to add parallelepipeds, which have proportions similar to those of the horse's body, just as the cube is similar to the lower part of the chair. Among other things, this tests the possibility that subjects identify the well-known cube geometry in a different way than they identify the geometry of similar, but less familiar objects.

\subsection{The Billboard Transformation}

To ensure that the billboard rotated objects did not stand out extensively by having different orientations than the $3 \mathrm{D}$ rotations we used the following procedure for rotating the billboard. The total rotation was broken into two parts, the second of which was the billboard rotation. The sum of the two parts was the total rotation. Thus the object to be placed on the billboard was rotated by the first part, after which it was projected onto the billboard. The billboard was then rotated by the second part. For example, when the rotation angle was $\theta$, and the billboard rotation angle $\phi$, the object was $3 \mathrm{D}$ rotated by $\theta-\phi$, then projected onto the billboard, which was rotated by $\phi$.

A simple case of the billboard transformation on a cube is shown in Figure 3. To the left the intermediate and the final views of a billboard rotated cube are shown; to the right a diagram presents a plan view of the cube and the billboard transformations. To scale, the view point is about $70 \mathrm{~cm}$ from the centre of the cube, so orthogonal projection was adequate for creating the diagram. Figure 3 shows the transformation where the final rotation, $\theta$ is $0^{\circ}$, and the 


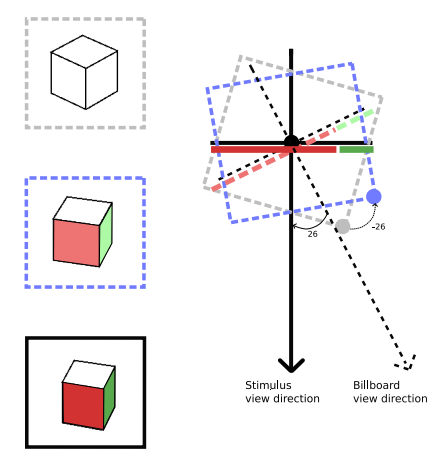

Figure 3: The left images are intermediate and final views of the cube: the top image is the cube projected from its canonical view; the middle image is the rotated cube projected onto the billboard, which is viewed face on; the lower image is the rotated billboard projected onto the view plane. On the right, the diagram shows a plan view of the cube and billboard with the two view directions, the one used to create the billboard image and the one used to project the rotated billboard onto the view plane. Seen from the billboard view direction, the grey square represents the top face in its canonical orientation, and the blue square represents the top face rotated by $-26^{\circ}$ to compensate for the billboard rotation. The blue cube is projected from the billboard view direction onto the billboard, with the dashed red and green lines representing the width of the two visible faces. Finally the billboard is projected onto the view plane from the stimulus view direction, showing the final width of the two visible faces of the cube in red and green solid lines.

billboard rotation, $\phi$, is $26^{\circ}$. First the billboard image is a central projection of a $3 \mathrm{D}$ cube rotated by $\theta-\phi=-26^{\circ}$ from its canonical orientation. Then, the billboard is rotated by $\phi=26^{\circ}$ and projected onto the view plane. The final image does not appear cubic, but too tall and narrow, as observed in the experiment.

The billboard rotation angles used in the experiment were $0^{\circ}, 15^{\circ}$, $-20^{\circ}, 26^{\circ},-32^{\circ}$ and $45^{\circ} .0^{\circ}$ was included because for that rotation billboard and 3D rotations are exactly the same, so that it acts as a check on the experiment implementation. Our preliminary experiment [Fourquet et al. 2006] showed that angles less than $15^{\circ}$ are indistinguishable from $0^{\circ}$, so we excluded small angles, concentrating the subjects' time on values where detectable distortion is known to vary. We used a combination of negative and positive angles because they show a clear signature of asymmetry between the two possible directions of rotation. For sufficiently symmetrical objects there is no difference between the directions and the set of angles produces a smooth curve. But both the horse and the chair have only approximate symmetries, and we wished to know if the broken symmetry would be significant.

\subsection{Participants}

The subjects were recruited informally from staff, graduate students and undergraduate students at the University of Waterloo; they were paid $\$ 10$ for participating. In total fourteen participants did the experiment, six in the first part and eight in the second part with the parallelepiped added, their ages ranged from 21 to 41 . Those who had significant artistic or computer graphics experience were identified so that possible differences between those subjects and the general population could be identified.

\subsection{Experiment Design}

The experiment used a complete factorial design: 4 object types, crossed with 3 billboard positions, crossed with 6 orientation orders among the three positions, crossed with 6 billboard rotations, to give 432 trials. Every subject did a complete set of trials.

\subsection{Procedure}

Every subject did their observations in a single session lasting roughly an hour. Each session began with ten practice trials, overseen by the experimenter, followed by any necessary clarification of the instructions.

The experiment proper consisted of 432 trials, divided into four blocks of 108 trials. Between blocks subjects were allowed to rest for as long as they wished. Subjects commented that they found the experiment easy but repetitive, which was expected. Fatigue was not a factor.

\subsection{Data Analysis}

The data was analysed using DataDesk ${ }^{T M}$, an interactive statistical analysis program, mainly using analysis of variance. Describing the results of the analysis we use the terminology, which is common in psychology: 'highly significant' when $p<0.01$, 'significant' when $p<0.05$, and 'marginally significant' when $p<0.10$.

We used a high threshold theory to measure response biases for different orientations. If the canonical view does indeed, make an object look more natural, i.e. less distorted and if we have chosen appropriate canonical views, then when subjects guess they will be least likely to guess the image with an orientation of $0^{\circ}$. To find out we ask the question, 'When subjects guess, are they more likely to guess one object rotation than another?' To answer, notice that each stimulus contains three target rotations, $-30^{\circ}, 0^{\circ}$ and $30^{\circ}$. High threshold theory provides the probability that rotation $k$ is chosen when guessing: when subjects see the distortion, which occurs with probability $p_{s}$, then they answer correctly; otherwise they guess rotation $k$ with probability $p_{k}$. The probability of answering $j$ when $i$ is the correct answer is then $\mu_{i j}=p_{s} \delta_{i j}+\left(1-p_{s}\right) p_{j}$, where $\delta_{i j}$ is the Kronecker delta, 1 when its arguments are equal, and 0 otherwise. The normalized contingency table, which gives the proportion of times that the subject responded $j$ when the correct response was $i, m_{i j}$ is then used to estimate the parameters of the model, $p_{s}$ and the three $p_{k}$ using ordinary least squares minimization. The solutions are simple: $p_{s}=\left(m_{11}+m_{22}+m_{33}-1\right) / 2$ and $p_{k}=\left(m_{1 k}+m_{2 k}+m_{3 k}-p_{s}\right) / 3\left(1-p_{s}\right)$. The interesting results we achieved using this technique are discussed below.

To make the graphs (Figures 4 to 6) more easily comparable to the response bias calculation, we have adjusted fraction correct for guessing using the high threshold theory described above.

\section{Experiment Results}

We started by comparing the data sets from the two parts of the experiment, finding no differences between the data from each. Consequently, we merged the two data sets for the remainder of the analysis.

We began by doing ANOVAs testing effects of subject. There was a significant difference, but only in overall levels of performance, and there was no indication that the subjects should be segregated into groups. Therefore, in the remainder of the analysis subjects were pooled. 

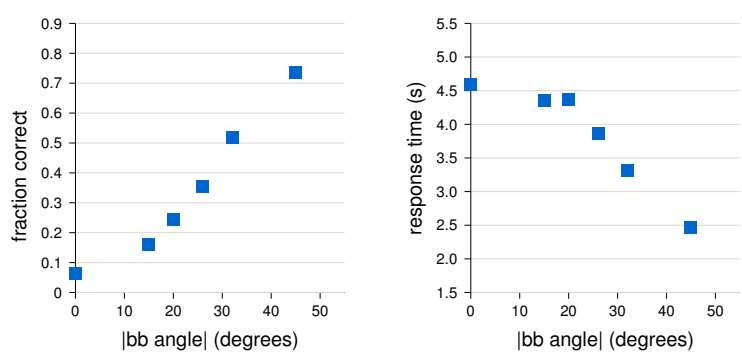

Figure 4: On the left: fraction correct according to billboard angles. On the right: response time according to billboard angles

The four graphs, shown in Figures 4, 5 and 6, illustrate the main results of our analysis: Figure 4 shows averages over all objects; Figures 5 and 6 segregate the data by object. Finally, an analysis of preferred view, using the analysis described in Section 3.7, is also presented.

\subsection{Results Common to all Objects}

The left graph of Figure 4 shows the fraction of trials in which the rotated billboard was correctly identified, graphed against the absolute value of the angle through which it was rotated. Subjects' ability correctly to detect distortion increases with billboard rotation angle. An ANOVA shows the main effect of billboard rotation to be highly significant $(F(5,6042)=123.39, p<0.0001)$. The check case has no billboard rotation, $\phi=0^{\circ}$, and the fraction correct is not significantly different from zero. Performance at the smallest non-zero billboard rotation $\left(\phi=15^{\circ}\right)$ is little different from no rotation. According to a Scheffé post hoc test, the difference is only marginally significant $(p=0.095)$. At larger rotation angles the difference from no rotation is highly significant. These results are exactly as expected: small billboard rotations create distortion that is imperceptible, but the distortion from large billboard rotations is easily visible.

The right graph of Figure 4 shows the average response time, also graphed against the absolute value of the billboard rotation angle. Subjects take longer to answer when the angle is small: an ANOVA shows this effect to be highly significant $(F(5,6042)=$ $61.464, p<0.0001$ ). Scheffé post hoc tests show the shorter response times to be highly significant for rotation angles above $20^{\circ}$. Notice that as fraction correct increases the response time falls, showing that the improvement in fraction correct is not the result of a speed-accuracy trade-off.

Our choice of billboard rotation angles was based on a pilot study and the results of Busey et al.[1990] that the distortion at less than $22^{\circ}$ of oblique viewing is subthreshold. We avoided rotation angles less than $15^{\circ}$, wishing to concentrate our data in the range where the distortion goes from scarcely perceptible to easily visible. Indeed, the analysis in Figure 4 shows that the region from $15^{\circ}$ to $40^{\circ}$ spans the transition between invisible and visible distortion. But the exact location of this region is relatively dependent on object properties as is shown in the next section.

\subsection{Differences between Objects}

In the analysis of variance there is no main effect of object, but there is a highly significant interaction between object and billboard rotation $(F(15,6042)=6.2726, p<0.0001)$. This effect is best seen by graphing the fraction correct against signed billboard angle. The graphs are shown in Figures 5 and 6.

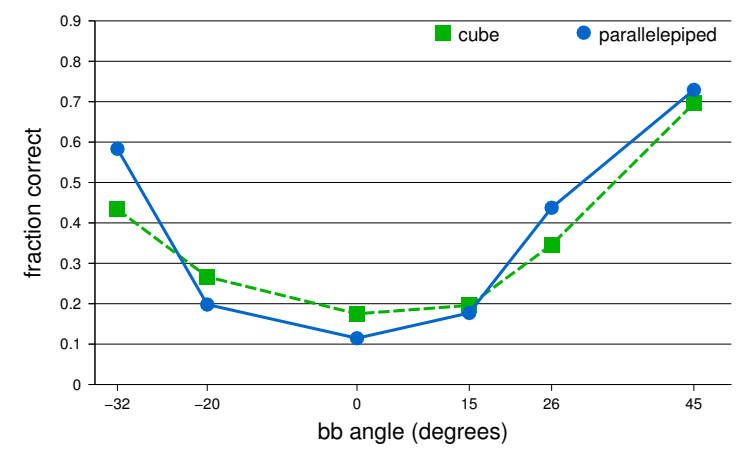

Figure 5: Fraction correct for each of the two geometric objects individually, the cube and the parallelepiped, according to billboard rotation angle.

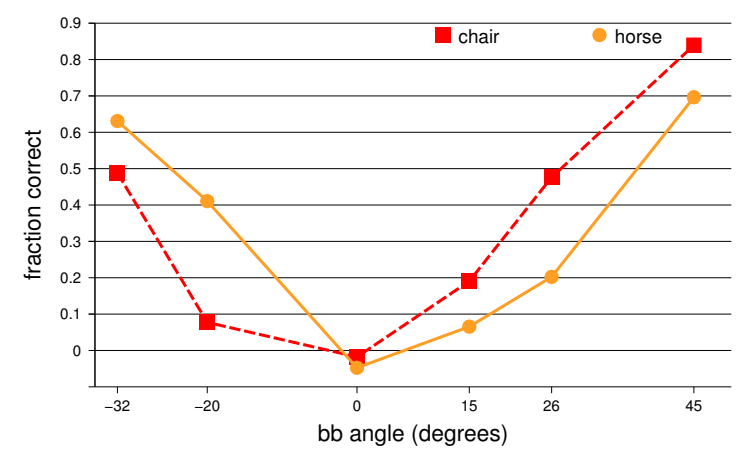

Figure 6: Fraction correct for each of the natural objects individually, the chair and the horse, according to billboard rotation angle.

The objects are grouped between the figures to show that they fall into two distinct groups: the geometric objects, cube and parallelepiped, show a pattern of fraction correct that is symmetric with respect to the sign of the billboard rotation; the natural objects, chair and horse, show a pattern that is asymmetric.

The geometric objects, Figure 5, have strong, easily visible rotation and reflection symmetries in 3D. Thus, the distortions caused by billboard rotation are symmetric with respect to the sign of the rotation and the resulting correctness curves are also symmetric. The two curves follow one another very closely, suggesting that distortions common to the two objects are most visible. However, such a conclusion accepts the null hypothesis, so more measurement is needed firmly to make such a conclusion.

The chair and the horse, Figure 6, the two curved objects lack 3D symmetries, so it is not surprising that correctness is asymmetric with respect to the direction of billboard rotation. The billboard horse appears more distorted when its head is facing the viewer; the chair seems more distorted when its seat back faces the viewer than when the chair is in profile. In Figure 7 these effects are shown in details for the horse. In the upper two rows the billboard orientation is $30^{\circ}$. At the top the billboard is on the right with the billboard rotation $\phi=-20^{\circ}$ and the distortion is strong. In the second row the billboard is in the middle with a larger billboard rotation, $\phi=26^{\circ}$. Yet the distortion is much weaker. Similarly, in the two lower rows, where the billboard orientation is $\theta=0^{\circ}$, distortion is clearly visible in the bottom row on the right where the billboard rotation is $\phi=-32^{\circ}$, and almost invisible in the row above where the billboard is in the middle with $\phi=26^{\circ}$. In both cases, the dis- 

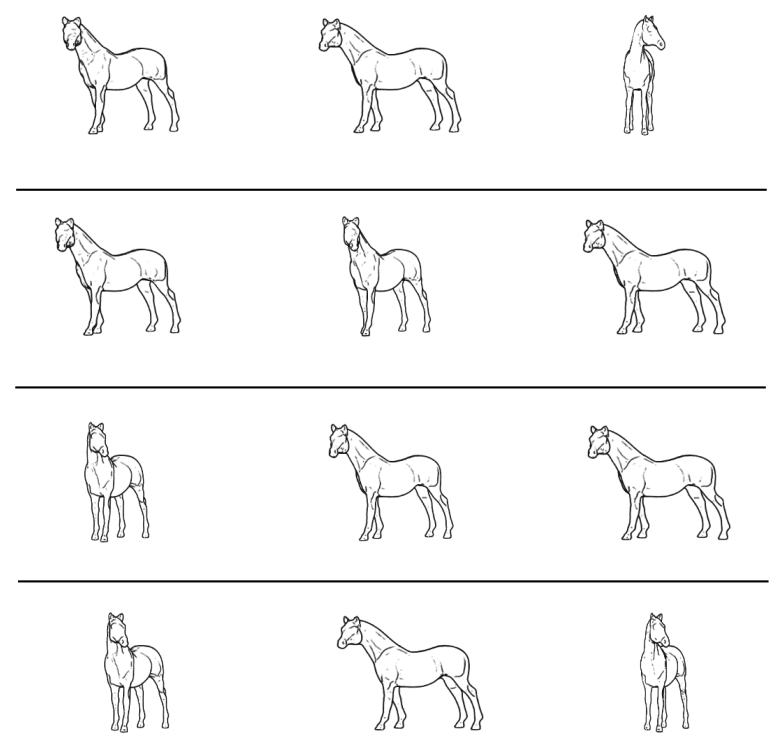

Figure 7: In the first two rows, the orientation is $\theta=30^{\circ}$ : in the first row, the billboard is on the right with a billboard rotation $\phi=-20^{\circ}$; in the second row, the billboard is in the middle with a billboard rotation $\phi=26^{\circ}$. In the last two rows, the orientation is $\theta=0^{\circ}$ : in the third row, the billboard is in the middle with a billboard rotation $\phi=26^{\circ}$; in the fourth row, the billboard is on the right with a billboard rotation $\phi=-32^{\circ}$.

tortion is strong when the horse body is hidden in depth, appearing as horizontal shrinking, and is weak when the horse body is almost parallel to the picture plane. The chair is shown in the first and third rows of Figure 2, both of which contain the billboard orientation $\theta=-30^{\circ}$. The distortion is more visible in the left image of the first row, where the billboard rotation is $\phi=15$ than it is in the left image of the third row, where the billboard rotation is larger, $\phi=-26^{\circ}$. For the chair the visible distortions for small rotation angles demand viewers' attention: there are not immediately visible, but can be seen on close inspection. The fraction correct is low, but the patterns in the data can be understood by examining such cases.

Figures 5 and 6 are averaged over three orientations while it is possible for the asymmetries to be concentrated in a single orientation, $-30^{\circ}, 0^{\circ}$ or $30^{\circ}$. In fact, the asymmetry is most pronounced for the horse when its orientation is $30^{\circ}$, and for the chair when its orientation is $-30^{\circ}$. As shown in Figure 7, the distortion of the horse at orientation $30^{\circ}$ is more asymmetric than at orientation $0^{\circ}$, since the distortions in the former are visible for a small negative billboard rotation, while being almost invisible for the $26^{\circ}$ rotation. For asymmetric objects billboard rotation looks better for specific orientations, which are not always the canonical view. However, there may be a method for choosing canonical views that are optimal for billboard rotation. To do so, we need to measure orientation preferences among billboard rotated objects and 3D rotated ones. In the next section we evaluate differences between two objects that are 3D rotated, and present at different orientations, to see if subjects are responding to inherent distortions of objects too close to degenerate orientations. The analysis presented there addresses this question, allowing us to ask if the preselected canonical views were optimal for billboard rotation.
Billboard Orientation

\begin{tabular}{lr|lll|r} 
& & $-30^{\circ}$ & $0^{\circ}$ & $30^{\circ}$ & $m_{i k}$ \\
\hline Selected & $-30^{\circ}$ & 0.62 & 0.21 & 0.26 & 1.09 \\
Orientation & $0^{\circ}$ & 0.14 & 0.51 & 0.19 & 0.84 \\
& $30^{\circ}$ & 0.24 & 0.28 & 0.55 & 1.07 \\
\hline & & 1.0 & 1.0 & 1.0 & $\sum m_{i i}=1.68$
\end{tabular}

Table 1: Contingency table from the normalized responses measured in the experiment for the orientation selected compared to the orientation of the billboard for the chair object

\begin{tabular}{|l|lllc|}
\hline & horse & chair & cube & parallelepiped \\
\hline$p_{\mathrm{s}}$ & 0.33 & 0.34 & 0.35 & 0.37 \\
$p_{-30}$ & 0.17 & 0.38 & 0.51 & 0.46 \\
$p_{0}$ & 0.35 & 0.25 & 0.17 & 0.19 \\
$p_{30}$ & 0.49 & 0.37 & 0.32 & 0.36 \\
\hline
\end{tabular}

Table 2: Probabilities of seeing the distorted billboard and probabilities of choosing the other orientations when the billboard is not present.

\subsection{The Impact of Orientation}

In order to investigate response biases to different orientations, we used the high threshold theory explained in Section 3.7. Table 1 shows the contingency table of orientation selected compared to billboard orientation for the chair as an example. (Tables for the other objects are similar.) From it, we could derive the probability that the distortion is seen, $p_{s}$, as well as the probability that an orientation is selected on guessing, $p_{-30}, p_{0}$, and $p_{30}$. The values for these probabilities are reported in the second column of Table 2, together with the probabilities for the other object.

In Table 1 the large diagonal values are the result of correct responses. The smaller off-diagonal elements - and part of the diagonal elements - are the result of guessing. Qualitatively, the centre row is guessed less often than the top or bottom rows, indicating the the canonical view chosen for the chair is indeed less likely to be seen as distorted when correctly projected.

Table 2 shows the calculated estimates for each object. The probability that distortion is seen, $p_{s}$, varies little from object to object: the standard deviation of each estimate is about 0.01 . This similarity is unsurprising because each estimate is averaged over the same set of angles, and because the detection probabilities are similar, as shown in Figures 5 and 6 . The estimates of responses bias, which are point estimates because the relevant sampling distribution is a Cauchy distribution, are more revealing.

The geometric objects, cube and parallelepiped, show strong avoidance of the canonical view. The chair shows less strong avoidance, indicating that a larger range of orientations is seen as canonical compared to the geometric objects. Presumably, the difference occurs because the chair's edges define its geometry less precisely. The horse has a qualitatively different pattern of response bias, with the $-30^{\circ}$ orientation strongly avoided. This orientation is shown in the right image of the to middle rows of Figure 7, where it can be contrasted with our chosen canonical view, left image in the second row. Either the assumptions of Section 3.7 are incorrect, or the profile view of the horse is canonical, questioning the assumed relationship between canonical and generic views. 


\section{Discussion}

Speaking broadly, the experiments described in this paper allow us to draw three conclusions. First, for objects seen from suitably canonical views, billboard rotation approximations to object rotation do not produce visible distortion for rotations less than $20^{\circ}$. What does this mean in practice? An image on a billboard is identical in its properties to an object with no depth. When an object with no depth is rotated two aspects of rotation occur: relative motion of its different parts, and foreshortening as parts of the object move towards the viewer and other parts away from the viewer. What does not occur is the appearance of previously invisible parts of the object, because all parts of a flat object can be seen at all rotations: from the front for $180^{\circ}$, from the back for the other $180^{\circ}$.

Now compare the flat object to a cube. As a cube is rotated every $90^{\circ}$ a new face comes into view. The face grows in size, then shrinks again, and $180^{\circ}$ later disappears. While the face is growing another visible face is shrinking; while the face is shrinking another visible face is growing. The appearance of the new face is a qualitative change; the change in relative size a quantitative one. The canonical view of a cube is far from orientations at which qualitative changes occur: the cube has two almost equally sized faces visible. When a picture of it rotates one of the faces comes toward the viewer, the other recedes. From foreshortening the receding face decreases in height; the oncoming face increases. These two effects are qualitatively similar to the $3 \mathrm{D}$ cube. But the oncoming face in the picture decreases in width, while the oncoming face of the $3 \mathrm{D}$ cube increases. The receding side decreases in width for picture and $3 \mathrm{D}$ cube. At $15^{\circ}$ of rotation the receding face of the cube has diminished in width by $30 \%$, the receding face of the picture by $4 \%$; the oncoming face of the cube has increased in width by $15 \%$, while the oncoming face in the picture has decreased by $4 \%$. It seems that the failure of the oncoming face to increase in size is the distortion noticed by our subjects, who mentioned that distorted cubes looked either too narrow or too tall.

In contrast to the cube every orientation of a sphere is canonical. When the sphere rotates its appearance does not change. But when a $2 \mathrm{D}$ projection of a sphere rotates it narrows. It is a general fact that pictures rotated about a vertical axis narrow. Among 3D objects narrowing on rotation is a sign of thinness. Introspecting, anomalous thinness is the giveaway of a distorted object. Thus, the surprise in this data is that $20^{\circ}$ seems a large threshold for visible distortion. The aspect ratio anomaly at this angle is about $25 \%$ for a cube, far above detection thresholds.

The second conclusion is that the geometric objects, the cube and the parallelepiped, are symmetrical with respect to the sign of the billboard rotation angle, while the more natural objects, the chair and the horse, are not. That is, in Figure 4 the two points with negative billboard rotations, $-20^{\circ}$ and $-32^{\circ}$, lie on the same line as the other points, showing strongly the symmetry of the overall data, but this simple curve is the average of three different curves. In Figure 5 the cube and parallelepiped are statistically indistinguishable, and symmetric with respect to the sign of the billboard rotation angle, and in Figure 6 the chair and horse are asymmetric, but with reversed sign, so that their sum is symmetric.

Looking more closely, we see that most of the horse asymmetry comes from final rotations of $30^{\circ}$, where the horse is seen head-on, and that most of the chair asymmetry comes from final rotations of $-30^{\circ}$, where the seat back of the chair is seen face on rather than in profile. In both cases a small billboard rotation in the wrong direction takes the object through a transition where qualitative features of the image change. The salient aspect of the horse is the angle between the head and the body. There is a qualitative change between the orientations where head and body point in different directions (top row right, second row center, third row left, and last row right and left in Figure 7) and where they point in the same direction (other positions in Figure 7). Both orientations look natural enough but when the billboard rotation takes the horse from one orientation to another (top and bottom right) the result is obviously distorted. For the chair similar effects occur when a billboard rotation goes from profile to facing.

The lesson is that the acceptability of geometric distortion depends strongly on particular features of the object, and that unpleasing distortion depends strongly on transitions to orientations in which identifiable features appear or disappear. This varies with object complexity in an interesting way. When the object is simple, with lots of symmetry, there are few such transitions and billboard rotation can be undertaken freely. When the object is complex, so much so that its feature complexity is textural and not a collection of individual features there are few such transitions. In this case also billboards can be rotated freely. But when the object is in the middle, with a substantial number of individually identifiable features there are many constraints on billboard rotation.

The third conclusion comes from the orientation preferences when guessing. Intuitively, an orientation that is guessed frequently is seen to be mildly distorted, even when it is not, and an orientation that is guessed infrequently is seen as being normal, perhaps typical. As Table 2 shows, $0^{\circ}$ orientations are least frequently guessed for the chair, cube and parallelepiped, indicating that our choice of canonical view was a good one for those objects. But for the horse, the orientation chosen least when guessing was $-30^{\circ}$, the top middle pose in Figure 7. Subjects obviously found this orientation most natural, possibly because it is common in children's picture books.

These three conclusions offer practical guidance for using rotated billboards as an inexpensive way of including complex content in 3D scenes. First we have identified a range of billboard rotations between $-20^{\circ}$ and $20^{\circ}$ that seems to be safe for most objects. This limit agrees with the results of Busey et al.[1990]. Second, we have identified the aspect of object orientation, the appearance of otherwise unseen surfaces, that is the fundamental limitation on billboard rotation. Third, we have discovered a new, and possibly more objective, method for testing canonical views.

In closing, we think that the experiment described in this paper shows how to push empirical investigation of perceptual acceptability deeper than is commonly done, past usability testing into the discovery and validation of rules and heuristics that can guide a wide range of research and implementation. It increases the range of applications for billboards, which is an advantage for many different parts of computer graphics, and it shows that canonical views need more investigation because they are bound to play a role in the perceptual evaluation of much computer graphics. To be sure, research on canonical views is time-consuming and demanding, but we think we have shown that there is promise to find widely applicable principles for defining and investigating canonical views. From the point of view of our long term objectives, this research is not the end, but only beginning the creation of a sound empirical foundation for our use of billboards as modelling primitives.

Acknowledgments The authors thank the reviewers for their comments. EF was financially supported by NSERC (PGSD3-3042292004). The horse mesh comes from The Large Geometric Models Archive at Georgia Institute of Technology, and the chair comes from the 3DTotal site and was created by Gerasidis Manolis, and is freely available for non-commercial use.

\section{References}

Blanz, V., TARR, M. J., AND BÜLthofF, H. H. 1999. What ob- 
ject attributes determine canonical views? Perception 28, 575599.

Busey, T. A., Brady, N. P., And Cutting, J. E. 1990. Compensation is unnecessary for the perception of faces in slanted pictures. Perception and Psychophysics 48, 1-11.

Cutting, J. E. 1987. Rigidity in cinema seen from the front row, side aisle. Journal of Experimental Psychology: Human Perception Performance 13, 323-334.

DeCarlo, D., Finkelstein, A., Rusinkiewicz, S., And SANTELla, A. 2003. Suggestive contours for conveying shape. ACM Transactions on Graphics (Proc. SIGGRAPH) 22, 3, 848855.

Décoret, X., Durand, F., Sillion, F. X., And Dorsey, J. 2003. Billboard clouds for extreme model simplification. ACM Transactions on Graphics (Proc. SIGGRAPH) 22, 3, 689-696.

Fourquet, E., Cowan, W., And Mann, S. 2006. Multiple perspectives in computer graphics: arguments from perceptual grouping and renaissance art. In $A P G V$ '06: Proceedings of the 3rd Symposium on Applied Perception in Graphics and Visualization, ACM Press, New York, 154-154.

Hamill, J., McDonnell, R., DobByn, S., AND O'Sullivan, C. 2005. Perceptual Evaluation of Impostor Representations for Virtual Humans and Buildings. Computer Graphics Forum 24, $3,623-633$

Jeschke, S., Wimmer, M., And Purgathofer, W. 2005. Image-based representations for accelerated rendering of complex scenes. In STAR Proceedings of Eurographics 2005, Eurographics Association, Dublin, Ireland, Y. Chrysanthou and M. Magnor, Eds., 1-20.

Maciel, P. W. C., ANd Shirley, P. 1995. Visual navigation of large environments using textured clusters. In SI3D '95: Proceedings of the 1995 Symposium on Interactive 3D Graphics, ACM Press, New York, 95-102.

Macmillan, N. A., And Creelman, C. D. 1991. Detection theory: A User's Guide. Cambridge University Press.

Palmer, S. E., Rosch, E., And Chase, P. 1981. Canonical perspective and the perception of objects. In Attention and Performance, IX, J. Long and A. Baddeley, Eds., 135-151.

Vishwanath, D., Girshick, A. R., And Banks, M. S. 2005. Why pictures look right when viewed from the wrong place. $\mathrm{Na}$ ture Neuroscience 8, 10, 1401-1410. 